\title{
Perceptually driven movements as contextual retrieval cues
}

\author{
MARGARET M. BRADLEY, BRUCE N. CUTHBERT, and PETER J. LANG \\ University of Florida, Gainesville, Florida
}

\begin{abstract}
Items were learned in the context of either a horizontal or a vertical eye movement, and later were retrieved in the context of the same or a different eye movement. The effect of a match in eye movement was measured for both perceptual processing and episodic recognition. Results indicate that the same eye movement significantly facilitated recognition speed of learned items, relative to recognition speed of items retrieved with a different eye movement. These data suggest that bodily information may be a salient dimension of the information represented in memory for an event, and that it can mediate context-dependent memory in other areas of experimental interest.
\end{abstract}

Investigations of cue-dependent memory performance have tended to neglect one class of potentially powerful retrieval cues: those that relate to the bodily responses that operate during learning and retrieval. The bioinformational theory of memory developed by Land (1979, 1984) directly addresses this deficiency, suggesting that efferent programs that direct somatovisceral responses are represented in the memory for an event, associated with stimulus-related and conceptually related material. Information regarding these responses may be hypothesized to include motoric information associated with performing specific overt actions, as well as the physiology supporting these acts. Assuming that access to a memory representation depends upon a match between the cues available at retrieval and the information stored, memory performance will be facilitated if similar response sets are operating at learning and retrieval.

Past work (Cuthbert \& Lang, 1984) suggests that eye movements are encoded during learning of verbal stimuli. The present study sought to determine whether execution of the same eye movement at learning and at retrieval facilitates memory, relative to different eye movements at learning and retrieval.

In a series of experiments, Jacoby (1983) demonstrated that the influence of a specific cue on later performance can be detected as facilitation in perceptual processing of the stimulus, or in recognition judgments concerning its prior occurrence, or in both. The design of the present experiment allowed assessment of the effect of eye movements on both perceptual processing and on judgments of prior occurrence. It was hypothesized that making the same eye movement would facilitate both types of decisions.

This research was supported in part by National Institute of Mental Health Grants MH41950 and MH37757 to P. J. Lang and Grant MH09422 to M. M. Bradley. Requests for reprints should be addressed to Peter J. Lang, Box J-165, JHMHC, University of Florida, Gainesville, FL 32610.
In the present experiment, the subject viewed a moving display of briefly presented characters. The subject's task was to detect occasional letters in a numeric stream and to identify the word produced by the letters. The display was presented moving horizontally (back and forth across the screen) or vertically (up and down across the screen) at encoding. In a later retrieval phase, the subject was again presented moving displays and asked to identify the items. In addition, the subject made a speeded recognition decision regarding whether the item had been seen in the encoding phase. In this retrieval phase, the eye movement required for a specific old item was either the same as or different from that required at encoding.

If the same eye movement, in conjunction with initial letter information obtained from the display, activated the item's representation in memory, then information regarding the item would be fed, in a top-down manner, to the perceptual recognition process; that is, activation of the item's memory representation would aid in completing the word before the final letters arrived perceptually. If this is the case, identification performance for previously seen items requiring the same eye movement should be faster than identification performance for items requiring a different eye movement. Although Jacoby (1983) used perceptual identification of degraded whole displays (i.e., brief presentation of the whole item), the procedure used here is comparable in that facilitation of perceptual processing must be produced by the addition of information from the item's memory representation.

Following identification, the subject was required to respond as quickly as possible whether or not the item was presented during the encoding phase. If the eye movement were stored with the item during encoding, the same movement would act as a retrieval cue and facilitate decisions about prior occurrence. Thus, speed of recognition decisions should be faster for same than for different eye movement trials. 


\section{METHOD}

\section{Subjects}

Thirty students from the University of Florida introductory psychology course participated individually for course credit.

\section{Materials and Design}

Forty six-, seven-, and eight-letter abstract nouns served as the verbal stimuli, and the digits $0-9$ served as the numeric filler items. The subject's head was stabilized to avoid head movement throughout the experiment. The letters and numbers were presented, one at a time, on an Apple Ile CRT screen; each character was presented for $35 \mathrm{msec}$, separated by a 350 -msec intercharacter interval. Embedded in the series of numbers were letters, in the correct sequence, that made up a noun.

Twenty characters (numbers and letters) were presented in a single sweep across the system. When the moving display reached the end of the screen, it reversed direction and presented 20 characters moving in the opposite direction. Approximately half of the letters of a word were presented in one sweep from one end of the screen to the other. That is, for horizontal displays, half of the letters were presented moving left to right and half moving right to left. For vertical displays, half of the letters were displayed moving down the center of the screen and half displayed moving up the screen. Forty characters constituted one full presentation of a word, and required approximately $15 \mathrm{sec}$ to complete.

In the encoding phase, 10 words were presented moving in a horizontal direction and 10 in a vertical direction. The particular direction and specific word chosen for any trial was randomized for each subject.

In the retrieval phase, the original 20 words were re-presented, with 10 of the 20 words displayed moving in the same direction and 10 words moving in a different direction. The factorial combination of eye movement at encoding and at retrieval produced four conditions, consisting of horizontal-horizontal, horizontal-vertical, vertical-vertical, and vertical-horizontal trials. In addition, 20 new words were presented, half displayed moving horizontally and half displayed moving vertically, randomly interspersed with the old words.

\section{Procedure}

In the encoding phase, the subject's only task was to detect the letters in the numeric display and identify the word formed by those letters. Each trial began with a tone signaling that the subject should get ready. The moving display began $2 \mathrm{sec}$ later. The subject continued viewing the display until the word was identified, at which time the subject pressed a button indicating identification, and then overtly articulated the item. The experimenter recorded errors.

Following the encoding phase, a 15-min retention interval occurred, during which time the subject completed several questionnaires.

In the retrieval phase, the subject again identified each item by pressing a button to terminate the display. In addition, immediately after the word was identified, the subject was instructed to indicate whether or not the item had been presented in the encoding phase by pressing a button labelled either "yes" or "no." Following this decision, the subject articulated the item. Speed and accuracy were stressed in the instructions. Identification time, recognition time, and recognition accuracy were the dependent measures.

\section{RESULTS}

It was hypothesized that words presented moving in the same direction at encoding and retrieval would be identified faster than words presented moving in different directions. Identification data are presented as the total number of characters (both letters and numbers) that the subject viewed before the item was identified. As a reference, $\mathbf{4 0}$ characters were viewed before all the letters of a word were presented once. The mean number of characters viewed for each of the four
Table 1

Perceptual Identification Performance (in Number of Characters Viewed), Recognition Reaction Time (in msec) and Hit Rate as a Function of the Eye Movement Occurring at Encoding and Retrieval

\begin{tabular}{|c|c|c|c|c|}
\hline \multicolumn{2}{|c|}{ Eye Movement } & \multirow{2}{*}{$\begin{array}{l}\text { Identification } \\
\text { Performance }\end{array}$} & \multicolumn{2}{|c|}{ Recognition } \\
\hline Encoding & Retrieval & & RT & Hits \\
\hline Horizontal & $\begin{array}{l}\text { Horizontal } \\
\text { Vertical }\end{array}$ & $\begin{array}{l}46 \\
46\end{array}$ & $\begin{array}{l}374 \\
429\end{array}$ & $\begin{array}{l}.82 \\
.82\end{array}$ \\
\hline Vertical & $\begin{array}{l}\text { Vertical } \\
\text { Horizontal }\end{array}$ & $\begin{array}{l}43 \\
44\end{array}$ & $\begin{array}{l}364 \\
451\end{array}$ & $\begin{array}{l}.88 \\
.82\end{array}$ \\
\hline
\end{tabular}

types of trials is presented in Table 1. Clearly, there were no differences in the number of characters viewed before correct identification occurred for same and different eye movement trials $(F<1)$.

The mean of 45 characters is close to the presentation rate of $\mathbf{4 0}$ characters per word, indicating that previously presented items were typically recognized with a single presentation of the item. This performance was significantly shorter than the performance for these same items in the encoding phase $[F(1,28)=65.69]$, in which horizontal trials required 66 characters and vertical trials required 65 characters for correct identification. In addition, identification times for old items were significantly shorter than for new items $[F(1,28)=78.11]$,with times of 60 and 58 characters for new items presented moving horizontally and vertically, respectively. Thus, although savings in perceptual identification occurred, there were no differential effects based on the direction of the eye movement.

However, eye movement clearly had an effect on the speed of recognition decisions. Table 1 illustrates the mean of the median reaction times for each condition in the retrieval phase. As is clear, recognition performance was facilitated when the eye movement was the same at encoding and retrieval $[F(1,28)=6.66, p=.015]$. For items initially presented moving vertically, there was an 86-msec advantage for items re-presented moving vertically over items re-presented moving horizontally. For items learned moving horizontally, there was a 56-msec advantage for items re-presented moving horizontally over items re-presented moving vertically. Recognition accuracy did not differ as a function of eye movement, with hit rates of .85 and .82 for same and different eye movement trials, respectively.

\section{DISCUSSION}

These data provide support for the assumption that memory representations code information regarding the somatic responses that occurred at input. The speed of recognition judgments was faster for items retrieved in the context of the same, rather than a different, eye movement.

However, no differences in the time taken to simply identify the item were apparent as a function of the eye movement occurring at encoding and retrieval. This suggests that the cuing provided by the same eye movement and the initial stimulus information (i.e., the first few letters of the item) did not result in activation of perceptual information (i.e., remaining letter information) that could aid in stimulus construction. 
However, it is possible that if the eye movement was not associated with multiple encoded items, such an effect might have been detected.

The role of the eye movement at retrieval appeared to be in contacting a specific episodic representation of the item, which allowed a faster decision regarding its prior occurrence. This finding suggests that a relatively neglected class of cues-those involving the bodily movements and responses that occur during encoding - may function to facilitate retrieval of specific events in memory.

The salience of a low-level nonspecific response cue, such as eye movement, in facilitating memory performance may seem counterintuitive. After all, eye movements are made constantly as the environment is scanned and apprehended, and such a cue would seem to be a prime candidate for overload (Watkins \& Watkins, 1975). However, the tracking movements required in this study are relatively unique, and do not resemble standard scanning movements. In addition, the task of visually tracking the moving display in this study was demanding and left neither time nor resources for elaborating other components of the encoded item (semantic, imaginal, etc.). Thus, in a sense, the only information represented in memory for the item may have been the letter information and the eye movement. In such an impoverished encoding environment, the eye movement made during encoding becomes a salient (perhaps the only) cue, and its ability to facilitate access to memory is apparent.

It is likely that cues that code information about bodily responses mediate retrieval performance in many other instances. Clearly, both stateand mood-dependent effects may be due to commonalities in the response sets that are engaged at learning and retrieval. At least one investigation of mood dependence (Bradley, York, \& Lang, 1987) demonstrated that this effect is obtained only when physiological responses are determined to be the same at learning and retrieval. Therefore, in addition to identifying a specific class of cues-those related to bodily responses-that operate at retrieval, the bioinformational approach has the added virtue of providing a methodology that is able to measure the presence or absence of relevant cues. By measuring physiological responses that occur during learning and recall, it is possible that heretofore invisible cues may become visible.

\section{REFERENCES}

Bradley, M. M., York, D. J., LANG, P. J. (1987). Emotion as context in memory. Psychophysiology, 24, 581. (Abstract)

Cuthbert, B. N., \&ANG, P. J. (1984). Eye movements in visual imagery. Psychophysiology, 21, 564. (Abstract)

JACOBY, L. (1983). Perceptual enhancement: Persistent effects of an experience. Journal of Experimental Psychology: Learning, Memory \& Cognition, 9, 21-38.

LANG, P. J. (1979). A bio-informational theory of emotional imagery. Psychophysiology, 16, 495-512.

LANG, P. J. (1984). Cognition in emotion: Concept and action. In C. Izard, J. Kagan, \& R. Zajonc (Eds.), Emotion, cognition and behavior (pp. 192-225). New York: Cambridge University Press.

Watkins, M. J., \& Watkins, O. C. (1975). Buildup of PI as a cue overload effect. Journal of Experimental Psychology: Human Learning \& Memory, 1, 442-452.

(Manuscript received April 12, 1988.) 